\title{
ON EQUATIONS OF THE WIENER-HOPF TYPE IN SEVERAL COMPLEX VARIABLES
}

\author{
EDGAR A. KRAUT
}

1. Introduction. In a previous communication [1] we established conditions under which the additive decomposition of a function of several complex variables is unique. In the analysis presented here we use the considerations of [1] to formulate a Wiener-Hopf problem in several complex variables which will be solved for a class of physically important kernels [2] by the method of successive approximations.

Statement of the Wiener-Hopf problem. Let $K\left(z_{1}, \cdots, z_{n}\right) \times$ $g_{1}\left(z_{1}, \cdots, z_{n}\right), z_{j}=x_{j}+i y_{j}$, be analytic in a tube $T:\left\{-\gamma_{i}<y_{i}<\delta_{i}\right.$, $\left.x_{i} \in(-\infty, \infty)\right\}$, and let $K$ have the form

$$
K\left(z_{1}, \cdots, z_{n}\right)=1-\lambda H\left(z_{1}, \cdots, z_{n}\right),
$$

where $\lambda$ is a complex parameter which may be made small and $H\left(z_{1}, \cdots, z_{n}\right)$ is a uniformly bounded analytic function in the tube $T$. Suppose that the $L_{2}$ norm

$$
\left\|g_{1}\right\|_{2}=\left\{\int_{-\infty}^{\infty} \cdots \int_{-\infty}^{\infty}\left|g_{1}\left(z_{1}, \cdots, z_{n}\right)\right|^{2} d x_{1} \cdots d x_{n}\right\}^{1 / 2}
$$

converges in $T$, then $\left\|K g_{1}\right\|_{2}$ also converges in $T$. Consequently, there exists in $T$ a unique additive decomposition $K g_{1}=\sum_{i=1}^{2^{n}} f_{i}$, where each $f_{i}$ is analytic and bounded in an octant shaped tube $T_{i}$ containing the interior of $T$, and $f_{i} \rightarrow 0$ as any one of the $y_{j} \rightarrow \pm \infty$ in $T_{i}$.

Given $f_{1}\left(z_{1}, \cdots, z_{n}\right)$ and the particular kernel $K\left(z_{1}, \cdots, z_{n}\right)$ in (1) determine the remaining $2^{n}$ unknown analytic functions $g_{1}, f_{2}, \cdots, f_{2^{n}}$ appearing in the decomposition of $K g_{1}$.

2. Solution of the Wiener-Hopf problem. Let the functions $g_{1}\left(z_{1}, \cdots, z_{n}\right)$ and $f_{1}\left(z_{1}, \cdots, z_{n}\right)$ be assumed to be analytic in the octant shaped tube $T_{1}: \operatorname{Im}\left(z_{i}\right)>0, i=1,2, \cdots$, and to have bounded $L_{2}$ norms in $T$. The decomposition of $K g_{1}$ has the form

$$
g_{1}-\lambda H g_{1}=\sum_{i=1}^{2^{n}} f_{i},
$$

and because this decomposition is unique, Cauchy integration yields

Received by the editors January 27, 1969. 
(3) $\quad+\frac{\lambda}{(2 \pi i)^{n}} \int_{-\infty}^{\infty} \cdots \int_{-\infty}^{\infty} \frac{H\left(\zeta_{1}, \cdots, \zeta_{n}\right) g_{1}\left(\zeta_{1}, \cdots, \zeta_{n}\right)}{\left(\zeta_{1}-z_{1}\right) \cdots\left(\zeta_{n}-z_{n}\right)} d \zeta_{1} \cdots d \zeta_{n}$, where $\operatorname{Im}\left(z_{1}\right)>0, \operatorname{Im}\left(z_{2}\right)>0, \cdots, \operatorname{Im}\left(z_{n}\right)>0$.

Equation (3) may be reduced to a singular integral equation for $g_{1}\left(z_{1}, \cdots, z_{n}\right)$ by allowing $\operatorname{Im}\left(z_{1}\right) \rightarrow 0^{+}, \cdots, \operatorname{Im}\left(z_{n}\right) \rightarrow 0^{+}$and introducing the generalized Plemelj formulas [3]. The result is

$$
g_{1}=f_{1}+\frac{\lambda}{2^{n}} \prod_{i=1}^{2^{n}}\left(I+S_{i}\right)\left(H g_{1}\right)
$$

where $I$ is the identity operator and where

$$
S_{i}=\frac{P}{\pi i} \int_{-\infty}^{\infty} \frac{d \zeta_{i}}{\left(\zeta_{i}-z_{i}\right)}
$$

are principal value integral operators. We state our main result for equation (4) as a

TheOREM. Let the maximum modulus of $H\left(z_{1}, \cdots, z_{n}\right)$ on $\operatorname{Im}\left(z_{i}\right)$ $=0, i=1,2, \cdots, n$ be denoted by $\max |H|$. Then for $0<|\lambda|<$ $(\max |H|)^{-1}$, and $g_{1}$ in the complete normed linear space $L_{2}$,

$$
T\left(g_{1}\right)=f_{1}+\frac{\lambda}{2^{n}} \prod_{i=1}^{2^{n}}\left(I+S_{i}\right)\left(H g_{1}\right)
$$

is a contraction mapping with respect to the $L_{2}$ norm. Hence the integral equation $T\left(g_{1}\right)=g_{1}$ has one and only one fixed point belonging to $L_{2}$. This fixed point is the limit of a sequence of successive approximations converging in $L_{2}$ norm to $g_{1}$.

Proof. Let $g_{1}$ and $h_{1}$ be members of the $L_{2}$ function space and analytic in $T_{1}$, we have

$$
\left\|T\left(g_{1}\right)-T\left(h_{1}\right)\right\|_{2}=\frac{|\lambda|}{2^{n}}\left\|\prod_{i=1}^{2^{n}}\left(I+S_{i}\right) H\left(g_{1}-h_{1}\right)\right\|_{2}
$$

Expanding the operator $\prod_{i=1}^{2^{n}}\left(I+S_{i}\right)$ and applying Minkowski's inequality to the result yields

$$
\left\|T\left(g_{1}\right)-T\left(h_{1}\right)\right\|_{2} \leqq|\lambda| \max |H|\left\|g_{1}-h_{1}\right\|_{2}
$$

where we have made use of the fact that the principal value operator $S_{i}$ gives the Hilbert transform, with respect to the $i$ th variable, of the 
function on which it operates and that the Hilbert transform is a bounded linear operator in $L_{2}$ satisfying [4] $\left\|S_{i} H g_{1}\right\|_{2}=\left\|H g_{1}\right\|_{2}$ and

$$
\left\|\prod_{i=1}^{2^{n}} S_{i} H g_{1}\right\|_{2}=\left\|H g_{1}\right\|_{2} .
$$

We have established that $T\left(g_{1}\right)$ is a contraction mapping with respect to the $L_{2}$ norm provided $|\lambda| \max |H|<1$. The remainder of the theorem is then a consequence of Banach's fixed point theorem [5].

Since we have now constructed a $g_{1}$ such that the conditions of Bochner's theorem [1] apply to the left-hand side of (2), the unknowns $f_{2}, f_{3}, \cdots, f_{2^{n}}$ in (2) are now uniquely determined by the Cauchy integral decomposition of $g_{1}-\lambda H g_{1}$. This solves the WienerHopf problem for kernels of the form indicated in (1).

\section{REFERENCES}

1. E. Kraut, S. Busenberg and W. Hall, On an additive decomposition of functions of several complex variables, Bull. Amer. Math. Soc. 74 (1968), 372-374.

2. E. Kraut and G. W. Lehman, Diffraction of electromagnetic waves by a righi angle dielectric wedge, J. Math. Phys. 10 (1969).

3. F. D. Gakhov, Boundary value problems, Pergamon Press, New York, 1966, pp. 70-72.

4. E. C. Titchmarsh, Introduction to the theory of Fourier integrals, 2nd ed., Clarendon Press, Oxford, 1948, p. 122.

5. W. Pogorzelski, Integral equations and their applications, Vol. I, Pergamon Press, New York, 1966, p. 197.

North American Rockwell Corporation, Thousand Oaks, California 